\title{
Bacterial Induction of Settlement and Metamorphosis in the Planula Larvae of Cassiopea andromeda (Cnidaria: Scyphozoa, Rhizostomeae)
}

\author{
R. Neumann
}

Institut für Entwicklungsphysiologie, Universität Köln, Gyrhofstraße 17, D-5000 Köln 41, Federal Republic of Germany

\begin{abstract}
Planula larvae were collected from mature Cassiopea andromeda medusae at Key Largo (Florida, USA). Their development was analysed and compared with the development of vegetative buds from laboratory-cultured polyps of this species. In the presence of substrate, settlement of the larvae, i.e. attachment and pedal disc formation, occurred within $24 \mathrm{~h}$; metamorphosis, i.e. elongation, segregation of stalk and calyx, and development of hypostome and tentacle anlagen, required another 2-3 days. Pedal-disc formation and metamorphosis - inhibited in sea water containing antibiotics - was shown to be initiated by substance(s) from marine bacteria of a standardized Vibrio sp. The inducing factor(s) is (are) released into the culture medium by bacteria growing in suspension cultures; centrifuged, non-growing bacteria cells, however, proved to be ineffective. Inductive capacity was detected in ultrafiltrates of suspension cultures, which included substances of low molecular weights of between 1,000 and 10,000 daltons. Following transverse bisection of the planula larvae, most of the apical fragments developed in sea water containing antibiotics - in the absence of metamorphosis-inducing factors - into polyp heads exhibiting neither stalk nor foot. Only a few fragments regenerated larvae of reduced size. In contrast, basal fragments quantitatively regenerated dwarf planulae capable of developing into complete polyps in the presence of substrate. These findings suggest that the basal pole of the larva directs both regeneration and head and stalk formation, the latter being under inhibitory control. When comparing the present results with those from observations of vegetative buds, striking similarities become apparent of developmental patterns and control mechanisms in scyphistoma morphogenesis, both in the generative and vegetative cycle.
\end{abstract}

\section{INTRODUCTION}

Cassiopea andromeda displays a metagenetic life cycle which is typical for the scyphozoans: scyphopolyps, representing the vegetative generation, produce medusae of separate sexes by means of monodiscous strobilation. Medusae reproduce sexually and eggs develop within the oral discs of the females; ciliated planula larvae are released, settle and undergo metamorphosis into scyphistomae. In addition, scyphistomae reproduce asexually by spindle shaped buds in the lower region of the polyp's head. After separation, the motile buds settle on suitable substrate and transform into polyps.

Bigelow (1900) dealt with the development of the buds up to the formation of medusae, in a detailed description of Cassiopea xamachana. Bud development under both normal and experimental conditions was analysed more recently in the same species by Curtis and Cowden (1971). Müller et al. (1976) and Hofmann et al. (1978) presented evidence that marine bacteria are involved in the control of polyp mor- phogenesis in buds of $C$. andromeda. The latter authors found that some heat-stable, dialyzable low molecular weight product(s) released into the medium by growing bacteria of a Vibrio sp. triggered bud metamorphosis.

Embryogenesis and scyphistoma development in rhizostomeae and in species of other scyphozoan families was studied as early as the last century and has led to the famous controversies on germ-layer problems between Claus, Goette, Hyde and others (see Berrill, 1949, for review). However, sexual reproduction, and embryonic and larval development of Cassiopea andromeda were only recently studied by Gohar and Eisawy (1960b) who also considered the question of fixation on the substrate and transformation of the larvae into scyphistomae.

The aim of the present work was to investigate mechanisms controlling settlement and metamorphosis in planula larvae of Cassiopea andromeda (syn.: C. xamachana) from Florida, USA. Experiments were performed to decide whether or not marine bacteria are as effective in promoting polyp formation in 
planulae as they are in buds of a laboratory-cultured strain of C. andromeda. Furthermore, dissection experiments were carried out in planulae. They were designed to investigate whether an inhibitory control of polyp formation by an endogenous inhibitor, which originates from the prospective foot region of the buds of C. andromeda (Neumann, 1977), is present also in the planula larvae.

\section{MATERIAL AND METHODS}

Fourteen medusae of the genus Cassiopea with umbrella diameters ranging from 4 to $13 \mathrm{~cm}$ were collected at the end of September, 1978, in a mangrove bay near Key Largo (Florida, USA). They lay in the typical position, with their oral arms upwards and their exumbrella on the muddy ground; this provided good camouflage considering the brownish-green colour of the animals. The oral arms measured significantly more than the umbrella radius and the medusae had a variable number of rhopalia (14-17). According to Mayer (1910), the medusae belong to $C$. xamachana Bigelow, 1892. In a study of the systematics of the genus Cassiopea, Gohar and Eisawy (1960a) concluded synonomy between $C$. andromeda, $C$. xamachana and some other closely related forms, with the exception of $C$. frondosa. For this reason, we have accepted the name of $C$. andromeda Forskal, 1775 as species name for our material.

Female medusae showed a yellowish area on the oral disc; this resulted from numerous vesicles, placed closely together. The same sexual characteristic was also observed earlier, by Bigelow (1893) and Smith
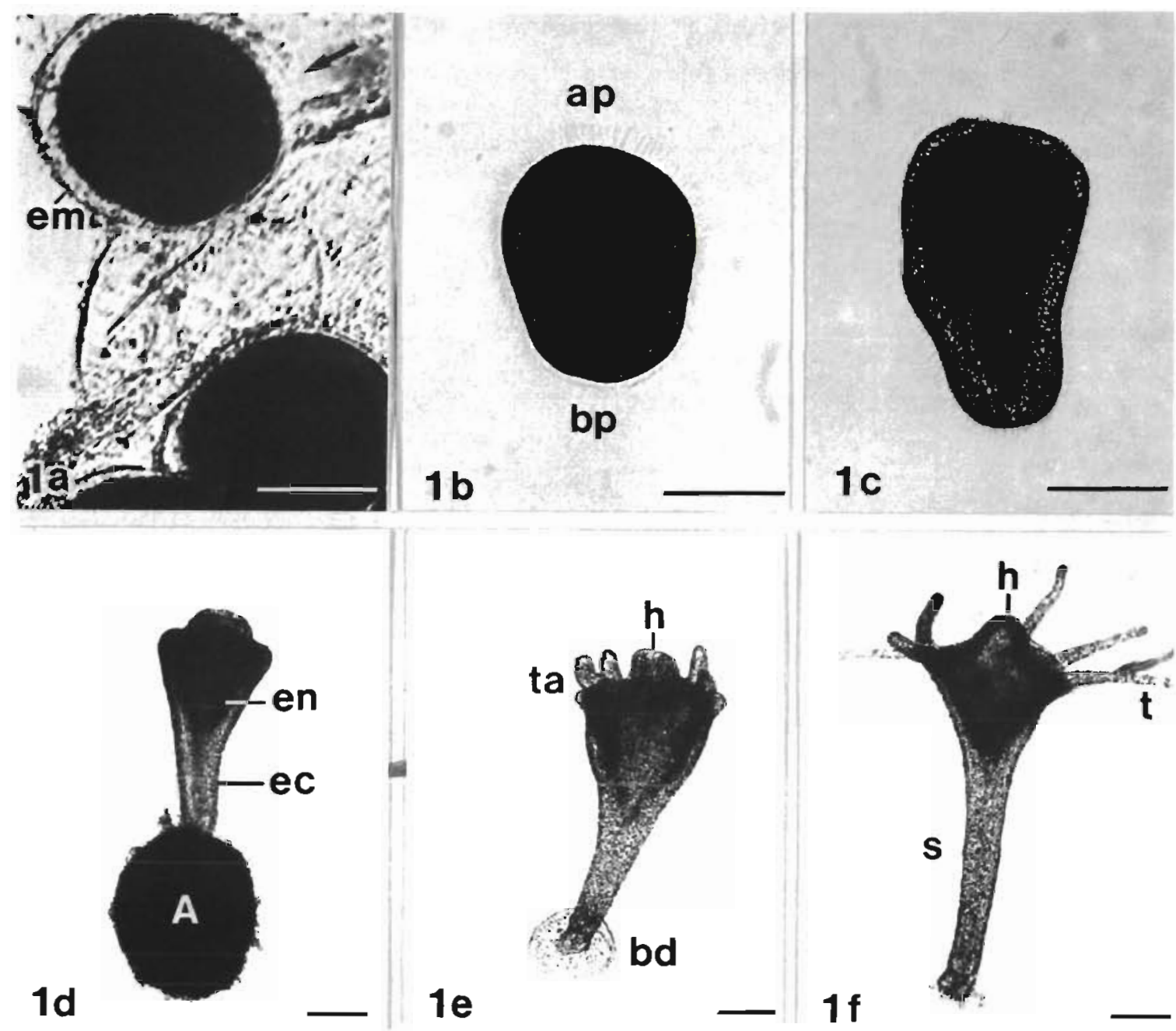

$1 e$
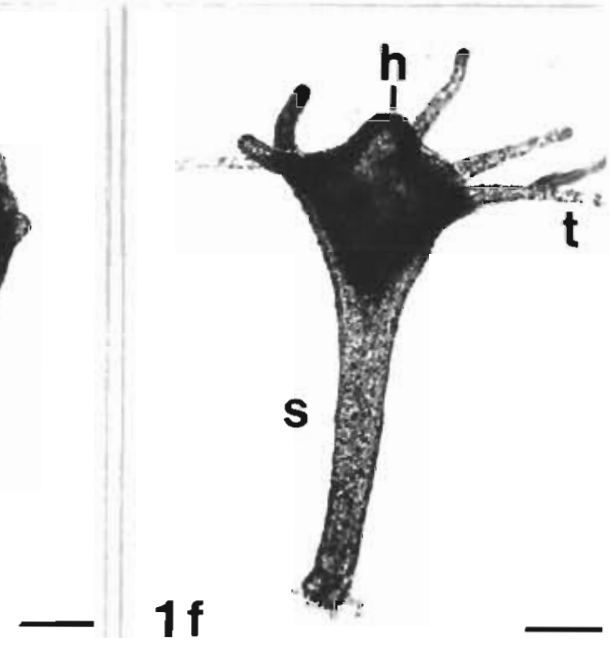

Fig. 1. Cassiopea andromeda (from Florida). Development of scyphopolyps from planula larvae. (a) Hatching of larva from egg membrane (arrow) and ambient gelatinous material; (b) hatched planula (note cilia); (c) 13-day old planula with elongated aboral end; (d) planula settled on an Artemia salina egg shell (A); (e) elongation (early metamorphosis) of the attached planula; (f) young scyphistoma. ap: apical pole; bd: basal disc; bp: basal pole; ec: ectoderm; em: egg membrane; en: entoderm; h: hypostome; s: stalk; t: tentacles; ta: tentacle anlagen. (All scale bars $=0.1 \mathrm{~mm}$ ) 
(1936), but not by Gothar and Eisawy (1960a). Embryonic and larval stages stuck in thick, gelatinous chains at the narrower bases of these vesicles.

Both hatched planulae and larvae still in their egg membranes were collected from the oral disc of the medusa. They were washed several times in sea water which had been sterilized with the aid of a filtersyringe (pore size of filter $0.22 \mu \mathrm{m}$ ). For transportation the larvae were transferred into sterile sea water to which was added $100 \mathrm{mg}$ penicillin- $\mathrm{K}$ and $129 \mathrm{mg}$ streptomycin-sulfate (Serva) per liter; several samples were placed into snap cap vials, the latter packed in two Dewar-boxes, and then dispatched.

On arrival in Cologne about 8 days after collection, the larvae were washed again and then stored at $21^{\circ}-23^{\circ} \mathrm{C}$ in Boveri dishes in about $150 \mathrm{ml}$ of pasteurized sea water containing antibiotics (see above).

Photographs were taken with a Zeiss photo-microscope. For statistics the fourfold $\mathrm{X}^{2}$-test was used.

\section{RESULTS}

\section{Hatching, Settlement and Metamorphosis of the Planulae}

When collected from the medusa, the planula larvae were, to a large extent, still in their egg membranes and thus attached to the vesicles of the oral disc, but a number of planulae were found swimming inbetween the vesicles. Shortly before hatching, the ciliated larvae began to rotate vigorously within their envelopes. On arrival in Cologne, almost all planulae had hatched from the membranes and the remaining individuals hatched within the next two days (Fig. 1). The planulae have an oval shape and measure about $200 \mu \mathrm{m}$ in length, i.e they are roughly a quarter the size of the vegetative buds of Cassiopea andromeda (Fig. 2). The future aboral (= basal) end, which is in front when swimming, can only be determined by its less blunted shape.

Settlement and metamorphosis began immediately after the planulae had been transferred into pasteurized sea water and provided with empty Artemia salina egg shells as a substrate. The larvae attached to the shells and formed pedal discs. The fixed larvae then elongated, divided into a foot area, a stalk and a calyx region, formed a hypostome and produced an increasing number of tentacle anlagen at the margin of the calyx. Throughout this paper the term 'settlement' designates attachment and pedal disc formation, and the dictional, but somewhat inaccurate term 'metamorphosis' stands for the subsequent stages of scyphistoma development.

The sequence of events in the polyp morphogenesis deriving from planulae is very similar to that of buds (Curtis and Cowden, 1971; Hofmann et al., 1978). However, the time required at $21^{\circ}$ to $23^{\circ} \mathrm{C}$ for the emergence of polyps with tentacles is quite different in the two cases: polyps from buds developed within about $24 \mathrm{~h}$, but planulae took about $24 \mathrm{~h}$ to settle and metamorphosis was not completed for another 2 days. A similar schedule of development in Cassiopea andromeda planulae was reported by Gohar and Eisawy (1960b).

Planulae which were continously cultured in sea water containing anitbiotics showed only limited morphogenesis: they usually developed a slightly elongated aboral end (Fig. 1c), small tentacle buds appeared in less than $1 \%$ of the larvae and hypostome forma-
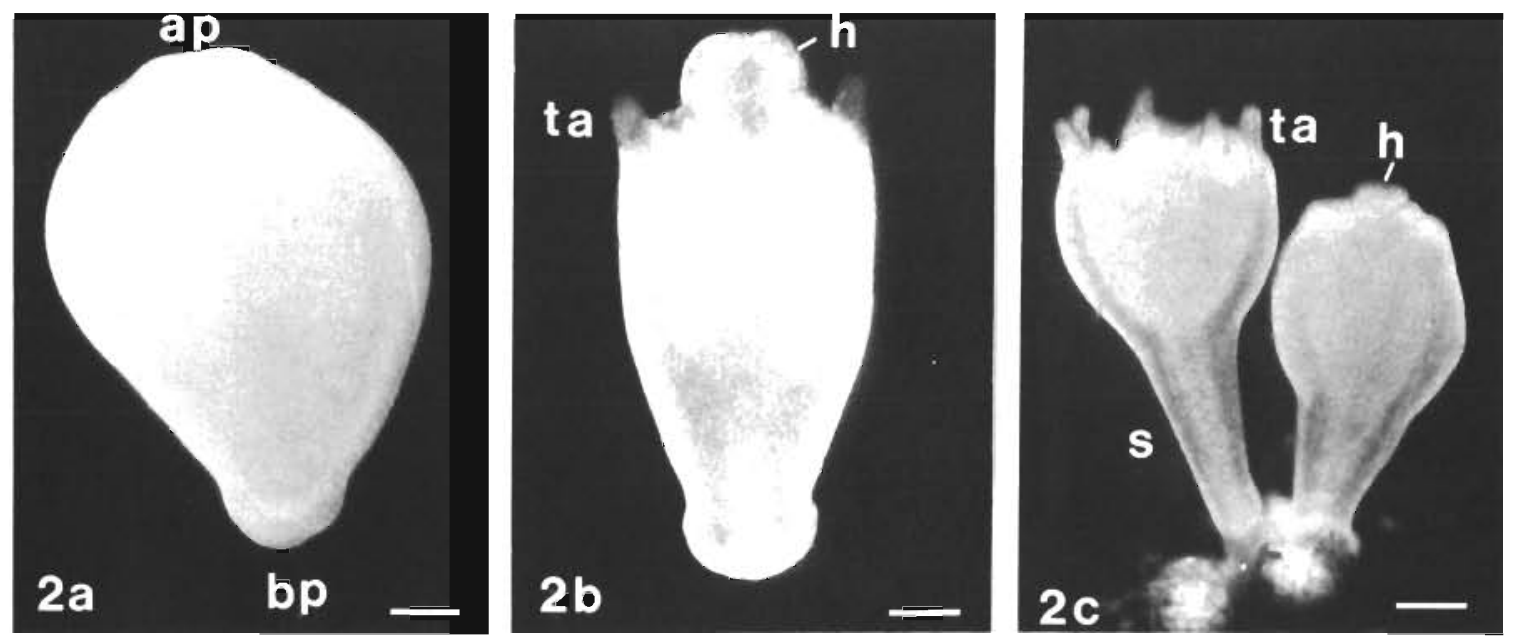

Fig. 2. Cassiopea andromeda (laboratory strain). Development of scyphopolyps from vegetative buds. (a) 3-day old bud; (b) swimming bud with hypostome and tentacle rudiments; (c) two buds attached to Artemia salina egg shells, at different stages of metamorphosis. For further explanations consult legend to Figure 1 
tion was an exception. None of these larvae settled. Furthermore, after 8 days of culture only 10 out of approximately 1,400 larvae had developed into polyps. Even planulae which had been in the sea water containing antibiotics for 27 days - approximately 100 in number - did not enter metamorphosis. Some of the larvae began to show degeneration after 3 weeks and were discarded.

\section{Bacterial Induction of Settlement and Metamorphosis}

In a series of preliminary experiments, planula larvae, previously cultured in sea water containing antibiotics for 9-16 days, were exposed to bacteria of a standardized, pure strain of a Vibrio sp., which were known to induce polyp morphogenesis in buds of Cassiopea andromeda (personal observation). Each group of 10 planula larvae was kept in a Boveri dish with $4 \mathrm{ml}$ of pasteurized sea water. Each of the following experiments was repeated three times; the observation period was 3 days.

Planulae showed no reaction when Vibrio sp. colonies, scratched from agar plates, were added to the larvae. Even after 3 days the planulae continued to swim around without any sign of metamorphosis.
When the planulae were placed together with a piece of agar (surface ca. $1 \mathrm{~cm}^{2}$ ) cut from a plate covered with bacteria colonies, the majority of larvae showed settling tendencies after $24 \mathrm{~h}$ and then started metamorphosis. Later, however, severe deformities occurred and polyp development failed to proceed any further. When the bacteria were grown in suspension cultures, separated by moderate centrifugation, and administered to the larvae, neither settlement nor metamorphosis was observed; after 3 days all planulae still continued to swim around. In contrast to this finding, after planulae were transferred to the supernatant of centrifuged suspension cultures, almost all of them began to settle within $24 \mathrm{~h}$ and started metamorphosis. Nevertheless, deformities appeared and polyp morphogenesis was interrupted, probably as a result of secondary bacterial growth occuring in the medium.

These observations indicated that bacteria of this Vibrio sp. could, to some extent, promote settling and metamorphosis in planulae. This effect was found, however, only when the larvae were either exposed to growing bacteria on agar or to supernatant of suspension cultures. Since these results agreed with earlier findings in bud metamorphosis (Hofmann et al., 1978), the subsequent experiments were focussed on bacterial products released into the ambient medium by growing bacteria.

Table 1. Cassiopea andromeda. Induction of settlement and metamorphosis in planulae, by bacterial products

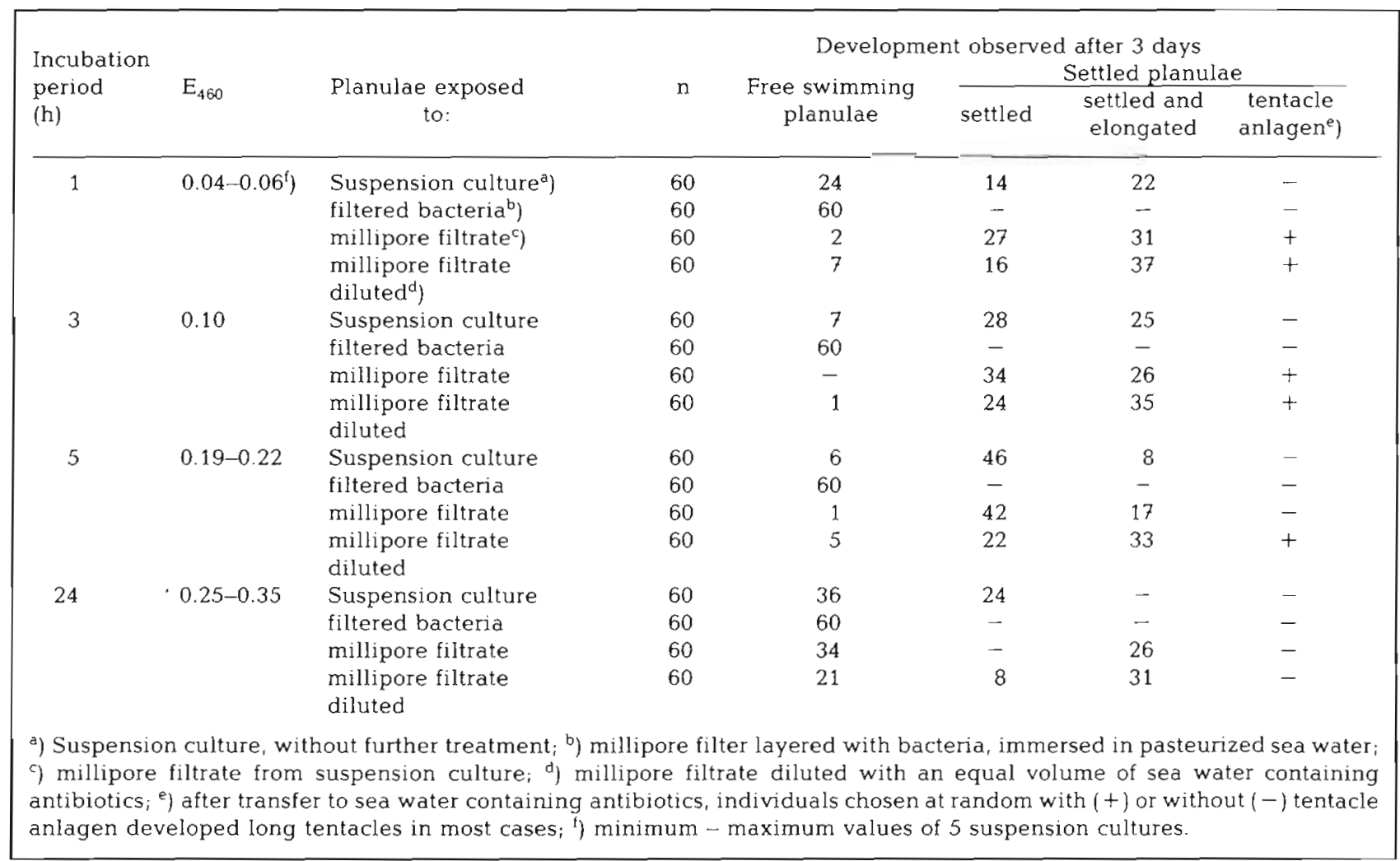




\section{Experiments with Bacteria Grown in Suspension Cultures}

The planula larvae were tested in several series of experiments with components of Vibrio sp. cultures grown suspended at $25^{\circ} \mathrm{C}$ in aerated nutrient solutions. The latter were prepared by dissolving either $0.1 \mathrm{~g}$ peptone (Difco), tryptic soy broth (Difco) or casein hydrolysate (Merck) in $500 \mathrm{ml}$ of natural sea water which was then autoclaved for $30 \mathrm{~min}$ at 0.5 bar. Bacteria, plated the day before on nutrient agar and incubated at $25^{\circ} \mathrm{C}$ were harvested and suspended; thereafter the nutrient solutions were inoculated and adjusted to the same bacteria titer.

After 1, 3, 5 and $24 \mathrm{~h}$ of incubation a photometric control was performed at $460 \mathrm{~nm}$ (red against distilled water), and the titer was determined by plating. Bacterial growth was similar in all cultures (footnote of Table 1). Samples were filtered using millipore filters, type GS $\varnothing 25 \mathrm{~mm}$, pore size $0.22 \mu \mathrm{m}$. The planulae were placed in groups of 6 per Boveri dish in $4 \mathrm{ml}$ of the respective test solution. No notable divergence was found in the reactions of the planulae to the bacteria grown in the three different types of nutrient solutions; hence the results obtained in 5 series of suspension cultures were summarized and presented in Table 1.

No significant differences in planula development were recorded in samples of Vibrio sp. cultures incubated for 1,3 or $5 \mathrm{~h}$ and containing $2 \times 10^{6}$ to $2 \times 10^{8}$ cells $\mathrm{ml}^{-1}$. In those samples of the suspension cultures, in which bacteria cells were always present, the planula larvae settled and began to metamorphose but deformities occurred frequently. Millipore filters layered with bacteria were ineffective in all cases, but millipore filtrates, either diluted or undiluted, proved effective - the larvae frequently settled on the glass wall of the Boveri dishes and began to metamorphose. Samples of bacteria cultures incubated for $24 \mathrm{~h}$ revealed a significant decrease in their capacity to induce polyp development and the planulae often became deformed.

In several cases the settled planulae remained in the initial spherical stage of metamorphosis, and in some dishes secondary bacterial growth occurred during prolonged observation. In an attempt to eliminate possible disturbing factors, a number of these larvae was carefully removed from the glass walls or from the water surface and placed in pasteurized sea water with or without antibiotics. The larvae now continued to metamorphose, developed a stalk and a calyx and in most cases formed long tentacles.

As a further step in analysing metamorphosis-inducing factors produced by Vibrio sp. cells, fractions of suspension cultures obtained by ultrafiltration procedures were tested with planula larvae.
Methods: Nutrient broth with $0.1 \mathrm{~g}$ casein hydrolysate $500 \mathrm{ml}^{-1}$ natural sea water was inoculated and incubated for about $3 \mathrm{~h}$. The bacteria were separated by millipore filtration when the suspension cultures had grown to a titer of $5 \times 10^{7}$ cells $\mathrm{ml}^{-1}$, equaling an $\mathrm{E}_{460}$ of 0.1 . Larger molecules were eliminated by ultrafiltration through membranes with an exclusion limit of 10,000 daltons (UM 10, Amicon) and afterwards the filtrate (which showed metamorphosis-inducing capacities when applied to buds) was run through a 1,000 daltons membrane (UM 2, Amicon). The residue (also found to be active in buds) with molecules ranging between 1,000 and 10,000 daltons was lyophilized. Because the number of planulae available was limited, the lyophilized sample was prepared in two dilutions of different concentrations and tested at $\mathrm{pH} 8.2$ and an osmolarity of $860 \mathrm{mOsmol}$ using a total of 60 larvae, 16 days after collection. In the first experiment the content of lyophilized material in the test solution was calculated to approximate the concentration existing in the native millipore filtrate; in the second experiment a two-fold concentration was applied. Virtually all larvae settled and started metamorphosis, some on the wall of the dishes and some hanging upside down at the surface. After 4 days nearly all individuals in the more concentrated test solution had elongated and formed tentacles, but a lower proportion of scyphistomae was recorded in the more dilute solution.

Ultrafiltered millipore filtrates from suspension cultures obviously permit metamorphosis of the larvae to proceed almost undisturbed. This is contrary to the effects of native filtrates, which were frequently found to block elongation and to inhibit tentacle growth in the settled planulae (Table 1).

\section{Development of Apical and Basal Fragments of Planulae}

Three samples with a total of 30 planulae which had been cultured for 18,22 or 25 days in sea water containing antibiotics were transversally bisected, according to their prospective significance, into apical and basal halves, using finest iridectomy scalpels. Their development was observed for 8 days.

A pical fragments: The results are presented in Fig. 3. They demonstrate that after healing, only a few apical halves resumed the organization of planulae. Almost all fragments transformed into polyp heads with neither stalk nor foot, within 8 days. In 3 cases the fragments appeared initially to regenerate the larval structure but later produced polyp heads instead.

Bas a l fragments: All basal halves of larvae restituted the larval organization and developed into smaller-sized planulae within 3 to 4 days. They never 


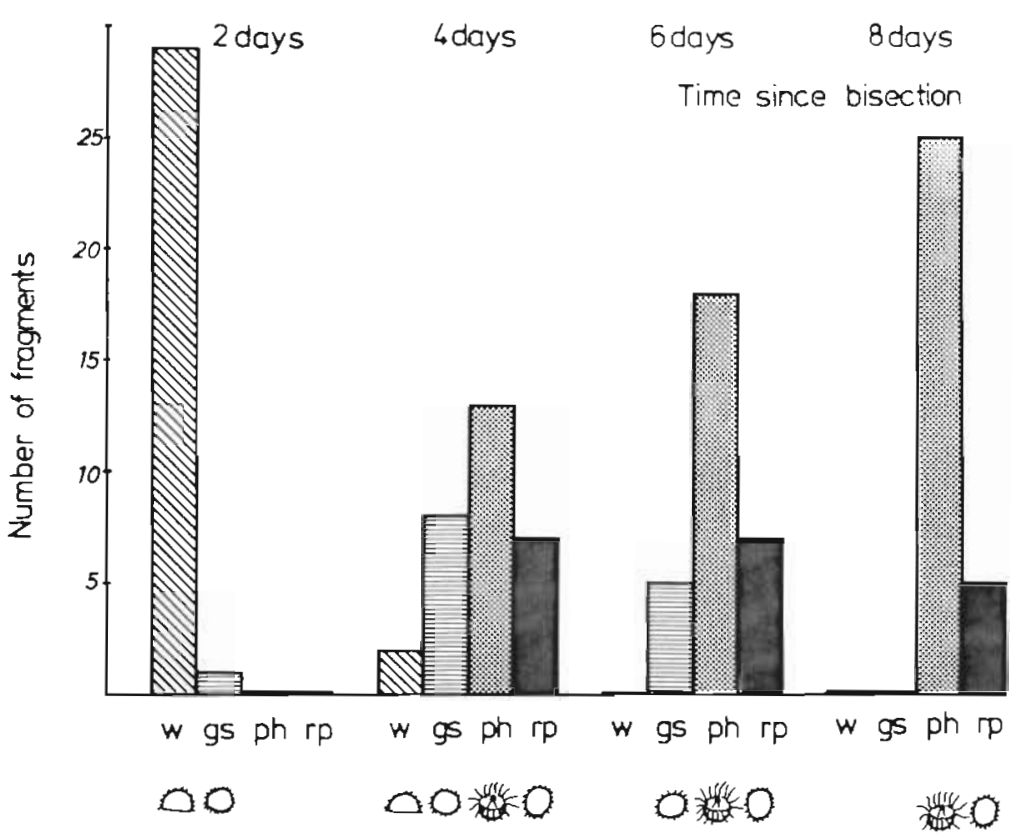

Fig. 3. Cassiopea andromeda. Development of apical fragments of planula larvae. Developmental stages: wound healing (w); globular undifferentiated stage (gs); polyp head without stalk of foot (ph); regenerated planula larva (rp)

produced solitary polyp heads as did the apical halves. The dwarf larvae could settle and metamorphose quite normally if substrate, e.g. Artemia salina egg shells, was added. In the latter case young scyphistomae with tentacles were recorded 7 days after dissection, at the earliest.

The results of these transection experiments on planulae agree with the observations of a (more extensive) series of similar experiments on vegetative buds of this species (Neumann, 1977). Both in planulae and in buds of Cassiopea andromeda, factors in the basal portion, representing the presumptive foot region of the polyp in each case, appear to favour regeneration of dwarf larvae (or dwarf buds) and also to exert an inhibitory control on the formation of stalk und calyx from the apical portion.

\section{DISCUSSION AND CONCLUSIONS}

Several authors have stated that both planula larvae and buds of Cassiopea species require a suitable substrate in order to settle. Gohar and Eisawy (1960b) provided the planulae of $C$. andromeda with small stones covered with paraffin wax mixed with charcoal. Curtis and Cowden (1971) emphasized the significance of an algal covering in the aquarium for the settling of $C$. Xamachana buds; they found that the buds would not attach to a clean glass surface.

In some marine invertebrates, bacteria were reported to account for settlement and/or metamorphosis of larvae; planula metamorphosis in the hydrozoan Hydractinia echinata represents the most thoroughly investigated case (Müller, 1969, 1973). Bacterial interference with polyp development from buds in Cassiopea species was mentioned more recently by Müller et al. (1976) and analyzed by Hofmann et al. (1978). Possible participation of bacteria in triggering settlement of planulae of Cyanea capillata was briefly considered by Brewer (1976). Metamorphosis is initiated by bacteria not only in Cnidaria but also in species of other phyla: this applies to metamorphosis of the pluteus larvae of the sea urchins Arbacia punctulata and Lytechinus pictus (Cameron and Hinegardner, 1974) as well as to the so-called catastrophic metamorphosis of Actinotrocha branchiata, the larva of the tentaculate Phoronis muelleri (Herrmann, 1976).

The basic mechanism of bacterial interaction with the larvae or buds of the above-mentioned species appears to be quite different to mechanisms present in Cassiopea andromeda. A pheromone-like, instable lipid, diffusing in very low quanitities from the outer layers of several species of marine bacteria, was found to guide metamorphosis in the planulae of Hydractinia echinata (Müller et al., 1976, and earlier papers). Herrmann (1976), however, believed electrokinetic phenomena - not necessarily linked with the excretion of tangible substances - to be responsible for metamorphosis in Actinotrocha branchiata. Transformation of both $H$. echinata and $A$. branchiata larvae can also be caused by some monovalent cations. Pluteus larvae of A. punctulata and $L$. pictus metamorphose in the presence of dialysable bacterial products with a molecular 
weight of less than 5,000 daltons (Cameron and Hinegardner, 1974).

Different modes of bacterial involvement in bud settlement and metamorphosis were found in the genus Cassiopea. According to Müller et al. (1976) the specific settling stimulus originates only from living bacteria in a sedentary state, grown as a stable film on horizontally-placed glass plates. No inducing activity was detected in the culture medium of the respective bacteria. Buds of $C$. andromeda settled and metamorphosed on glass slides covered with a heterogenous microbial layer. These plates had previously been placed in sea water used for incubation of Artemia salina eggs; and an effective strain of a Vibrio sp. could be isolated from the A. salina cultures (Hofmann et al., 1978). Contrary to the findings of the former authors, one or more factors causing rapid, synchronous settlement and polyp formation were detected in culture medium separated from log-phase suspension cultures of this Vibrio sp. The heat-stable, dialysable product was apparently not present when buds were exposed to bacterial layers of living, but non-growing, bacteria cells placed on millipore filters and immersed in pasteurized sea water.

As reported in the present paper, a standardized strain of a Vibrio sp. stimulated planulae of Cassiopea andromeda, obtained from Florida, to settle and to metamorphose; it stimulated also buds which had been exposed to identical preparations. Both buds and larvae reacted upon bacterial products of low molecular weight within the range of 1,000 to 10,000 daltons, released into the culture medium. However, in contrast to the experiments with planulae, buds of one batch showed always synchronous behavior and metamorphosed considerably faster.

Prolonged exposure to preparations with an inductive capacity frequently caused disturbance of scyphistomal development: growth of tentacles appeared most susceptible to detrimental effects. Replacement of these latter solutions by pasteurized seawater with or without antibiotics, after the planulae had formed a pedal disc, could successfully combat these problems; they are probably due to various metabolic by-products present in the preparations. This observation implies that, also in planula metamorphosis, the bacterial stimulus is required only for the initial phase of development, i.e. for a period of about $24 \mathrm{~h}$.

The behavior of oral and aboral bud halves was first described by Curtis and Cowden (1971) for Cassiopea xamachana. Müller et al. (1976) also noted that basal (= aboral) fragments of Cassiopea sp. buds can regenerate, whereas apical portions tend to transform into scyphistomae. An extensive series of experiments of the buds of $C$. andromeda (Neumann, 1977) showed that the differentiation and regeneration capacities of the fragments depend not only on the original position in the organism and on the size of the fragments, but also on the age of the dissected buds. A high percentage of young, small apical fragments produced polyp heads without stalk or foot, whereas apical fragments of older buds, and in particular those fragments comprising most of the bud tissue, revealed an increased tendency to regenerate the bud structure, as did all basal fragments irrespective of size and age.

The results of the present paper suggest that in both the planulae and the buds of Cassiopea andromeda the basal, presumptive foot region displays an inhibitory control on polyp morphogenesis, by suppressing premature metamorphosis of the larvae of larva-like buds. Factors originating from bacteria appear to be the specific stimuli which suspend the inhibitory mechanism and induce settlement and metamorphosis.

Acknowledgements. I should firstly like to thank Priv.Doz. Dr D. K. I Iofmann for reading the manuscript and for many valuable suggestions. I am also indebted to Dr C. R. Robins and Dr H. B. Michel, of the University of Miami, Florida, for use of optic facilities required during collection of the planulde, and to Prof. Dr. C. Harte for facilities in the Cologne institute. Finally, I wish to thank Mrs. Jenny Hoyt for her help with my English manuscript.

\section{LITERATURE CITED}

Berrill, N. J. (1949). Developmental analysis of Scyphomedusae. Biol. Rev. 24, 393-410.

Bigelow, R. P. (1893). Some observations on Polyclonia frondosa. Johns Hopkins Univ. Circ, 106, 106.

Bigelow, R. P. (1900). The anatomy and development of Cassiopea xamachana. Mem. Boston Soc. nat. Hist., 5 , $193-236$.

Brewer, R. H. (1976). Some microenvironmental influences on attachment behavior of the planulae of Cyanea capillata. In G. O. Mackie (Ed.), Coolenterate Ecology and Behavior. Plenum Press, New York. pp. 347-355.

Cameron, R. A. and Hinegardner, R. T. (1974). Initiation of metamorphosis in laboratory cultured sea urchins. Biol. Bull. mar. biol. Lab., Woods Hole, 146, 335-342.

Curtis, S. K. and Cowden, R. R. (1971). Normal and experimentally modified development in buds of Cassiopea. Acta Embryol. exp., 3, 239-259.

Gohar, H. A. F. and Eisawy, A. M. (1960a). The biology of Cassiopea (from the Red Sea) (with notes on the species problem). Publs mar biol. Stn Ghardaqua, 11, 5-42.

Gohar, H. A. F. and Eisawy, A. M. (1960b). The development of Cassiopea andromeda (Scyphomedusae). Publs mar. biol. Stn Ghardaqua, 11, 148-190.

Herrmann, K. (1976). Untersuchungen über Morphologie, Physiologie und Okologie der Metamorphose von Phoronis muelleri (Phoronidae). Zool. Jb. (Abt. Anat. Ontog. Tiere), 95, 354-426.

Hofmann, D. K., Neumann, R. and Henne, K. (1978). Strabilation, budding and initiation of scyphistoma morphogenesis in the Rhizostome Cassiopea andromeda (Cnidaria: Scyphozoa). Mar. Biol., 47, 161-176. 
Mayer, A. G. (1910). Medusae of the world. Publs Camegie Instn, 109, 636-650.

Müller, W. A. (1969). Auslösung der Metamorphose durch Bakterien bei den Larven von Hydractinia echinata. Zool. Jb. (Abt. Anat. Ontog. Tiere), 86, 84-95.

Müller, W. A. (1973). Metamorphose-Induktion bei Planulalarven. I. Der bakterielle Induktor. Wilhelm Roux Arch.EntwMech.Org., 173, 107-121.

Müller, W. A., Wieker, F. and Eiben, R. (1976). Larval adhesion, releasing stimuli and metamorphosis. In G. O.
Mackie (Ed.), Coelenterate Ecology and Behavior. Plenum Press, New York. pp. 339-346

Neumann, R. (1977). Polyp morphogenesis in a scyphozoan: evidence for a head inhibitor from the presumptive foot end in vegetative buds of Cassiopea andromeda. Wilhelm Roux Arch.EntwMech.Org., 183, 79-83.

Smith, H. G. (1936). Contribution to the anatomy and physiology of Cassiopea frondosa. (Publs. Carnegie Instn 475), Pap. Tortugas Lab., 31, 17-53.

This paper was submitted to the editor; it was accepted for printing on May 17, 1979 